Research Article

\title{
Study on Fluid-Structure Coupling Vibration of Compressor Pipeline
}

\author{
Jia Wu $\left(\mathbb{D},{ }^{1}\right.$ Chunjie Li $\left(\mathbb{D},{ }^{2}\right.$ Shuiying Zheng $\mathbb{D}^{2},{ }^{2}$ and Jingheng Gao ${ }^{3}{ }^{3}$ \\ ${ }^{1}$ School of Mechanical Engineering, Zhejiang University, Hangzhou 310027, China \\ ${ }^{2}$ Institute of Chemical Machinery, Zhejiang University, Hangzhou 310027, China \\ ${ }^{3}$ Hangzhou Jizhi Mechatronic Co., Ltd., Hangzhou 310030, China \\ Correspondence should be addressed to Shuiying Zheng; zhengshuiying@zju.edu.cn
}

Received 26 March 2019; Revised 17 June 2019; Accepted 17 July 2019; Published 7 August 2019

Academic Editor: Huu-Tai Thai

Copyright (C) 2019 Jia Wu et al. This is an open access article distributed under the Creative Commons Attribution License, which permits unrestricted use, distribution, and reproduction in any medium, provided the original work is properly cited.

\begin{abstract}
In practical engineering, pipeline vibration is often not caused by a single factor but by a combination of many factors. A fluidstructure coupling method is proposed in this paper and used to study the vibration of the compressor pipeline under the interaction of pipeline structure and airflow in it. The method is based on structured grids, so that the displacements of grid nodes can be calculated accurately at each time step. The results of transient calculation show that when the given inlet mass flow rate is constant and there is no other disturbance, the pressure fluctuation and the vibration of pipeline structure will occur by using fluid-structure coupling, and the vibration frequencies are consistent with the third- and fifth-order structural natural frequencies. Moreover, the higher the pressure in the pipe, the greater the fluid-structure coupling vibration. In addition, the fluid-structure coupling vibration not only occurs in the studied pipeline but also propagates to distant downstream pipeline. Comparing the above results with experimental results, it is found that the results of fluid-structure coupling calculation are in agreement with the actual situation, which shows that the method is reasonable and reliable and can be applied to engineering.
\end{abstract}

\section{Introduction}

Compressor pipeline vibration is a hidden danger that cannot be ignored for safe production of factories. Strong pipeline vibration affects the normal operation of devices and causes great harm. Many studies have been done on the vibration of compressor pipeline. They are mainly divided into three aspects.

Firstly, it is the study of airflow pulsation in the pipeline. The vibration of the compressor pipeline encountered in production is mostly caused by air pulsation, especially in reciprocating compressor. Reciprocating compressor is characterized by intermittent and periodic suction and exhaust, and thus it will stimulate the inlet and outlet of the pipeline to produce intense air pulsation, which has a serious impact on the performance and work. Only by thoroughly studying the mechanism of airflow pulsation, establishing a reasonable analysis model of airflow pulsation, accurately predicting the airflow pulsation in the compressor pipeline system, and reasonably designing the structural parameters of the pipeline, can the airflow pulsation and its influence be controlled in the minimum range. Skudrzyk [1], in order to study the fluid characteristics in the pipeline, used the plane wave theory to study the dynamic characteristics of the gas column. Hayama et al. [2] revised the assumption of plane wave theory of pipeline in resonance zone, which greatly reduced the relative error of the revised calculation results and basically met the design requirements of practical engineering. Maclaren et al. [3], considering environmental factors, proposed conservative and characteristic equations with more perfect mathematical models and more accurate calculation methods, which were suitable for one-dimensional unsteady flow and more close to the actual flow of gas in the pipeline. Durant et al. [4] carried out vibration measurement induced by airflow in a straight pipe and obtained the spectral density curve of air pulsation at different Mach numbers; the velocity spectral density of vibration and the sound pressure value radiated outward. In 
addition, the API Standard 618 of American Petroleum Institute specifies the design criteria of gas flow pulsation and pipeline vibration for reciprocating compressors used in the petrochemical industry.

Secondly, the dynamic characteristics and dynamic responses of pipeline structures are analyzed and studied. Practice shows that even though the airflow fluctuation in the pipeline has been controlled within a very small range, mechanical resonance can still be caused by the unreasonable design of the pipeline structure, resulting in strong vibration of the pipeline. Therefore, the structural dynamic characteristics of the pipeline must be carefully studied. Païdoussis and Issid [5] regarded the pipeline as a beam model and derived the motion equation. After considering the pipeline as a beam model, Rayleign method, Dunkerley method, Ritz method, and Galerkin method can be used to solve the low-order structural natural frequencies of simple pipeline. For pipelines with complex spatial structures, the low-order structural natural frequencies can be solved by the finite element method, transfer matrix method, and impedance analysis method [6]. Irie et al. [7] used the Timshenko beam model to derive the transfer matrix method for vibration and stability of conveying fluid pipeline. Lesmez et al. [8] had done pioneering work on the transfer matrix method for vibration of the space pipeline system. In the process of derivation, the method of separating variables is used, and the eigenvalue is included in the final characteristic matrix of the pipeline system.

Finally, the fluid-structure coupling vibration of the pipeline is studied. The fluid flow in the pipeline will lead to the vibration of the pipeline structure, and the vibration of the pipeline structure will in turn affect the motion state of the fluid in the pipeline. Therefore, coupling vibration between fluid and structure is produced in the pipeline. The fluid-structure coupling vibration has a great impact on the dynamic characteristics of the pipeline. Wiggert et al. $[9,10]$ established 4-equation model and 14-equation model for the study of fluid-structure interaction vibration of straight pipes. Erath et al. [11] studied the effect of water hammer with fluid-structure interaction on the vibration response and characteristics of pipe systems. Tijsseling and Lavooij [12] used the characteristic line method to analyze the dynamic response of the pipeline system under the action of the water hammer. The characteristic line method is suitable for the simple pipeline system. However, for the complex pipeline system, the finite element method has high accuracy and efficiency. Sreejith et al. [13] used the finite element method to analyze the fluid-structure coupling dynamic response of pipeline under variable velocity. Pittard et al. [14] used FLUENT software to establish a large eddy model and studied the influence of fluid pressure fluctuation on pipeline vibration characteristics. Menter et al. [15] used ANSYS MFX fluid-structure multiphysical field coupling scheme based on elastic dynamic mesh deformation technology to calculate the finite length elastic tube. The calculated vibration pattern and the discharge of the lagging vortices of the elastic tube agreed well with the theoretical structure. In addition, many experiments have been carried out to revise and validate the fluid-structure coupling theory. Vardy et al. [16-18] studied the coupled vibration of branched pipes, valves, 90 degree curved pipes, and single-elbow pipe systems by means of experiments. Ziada et al. [19] carried out an experimental comparative study on the fluid-acoustic coupling theory of T-shaped pipes. Duan et al. [20] used experiments to study Y-shaped pipes similar to T-shaped pipes, found the gas-solid twophase flow law in this type of pipes, and obtained valuable experimental data.

The purpose of this paper is to study the vibration of the compressor pipeline under the interaction of pipeline structure and airflow by using a proposed fluid-structure coupling method and then to verify the effectiveness of this method by comparing with experimental results.

\section{Fluid-Structure Coupling Method}

To simulate the transient calculation of pipeline vibration, a fluid-structure coupling method combining three-dimensional transient flow field simulation with structural dynamic calculation is proposed. Transient flow field simulation is solved with the mesh movement method. The motion equation used in structural dynamic calculation adopts the modal order reduction method. The data exchange between fluid dynamics and structural dynamics is realized by data files, which makes quasicoupling calculation possible. The fluid-structure coupling is described by the weak coupling method. This means that the fluid and structural equations are computed in every time step, and the data transferred at the fluid-structural interface are used as the boundary condition of the two domains. One advantage of the weak coupling method is to make full use of the mature computational fluid dynamics (CFD) software without rewriting the code. The flow chart of fluid-structure coupling transient calculation for pipeline vibration is shown in Figure 1.

The transient flow field of pipeline is simulated by FLUENT software. The fluid forces are obtained by integrating the fluid pressures through the application of userdefined function (UDF). With the fluid boundary condition transferred to a data file, the displacement is obtained by solving the motion equation. After the structural boundary condition is transferred to the data file, the CFD code is then applied to update the grid position based on the proposed mesh movement method. This iterative procedure is repeated until the vibration curve is stable.

2.1. Modal Order Reduction. Modal order reduction is to replace the original complex high-order system model with a low-order model which can maintain the main characteristics of the original system. It is required that the results of the low-order model can be close to those of the original system.

The motion equation of the pipeline system is

$$
[M]\{\ddot{q}\}+[C]\{\dot{q}\}+[K]\{q\}=\{f\},
$$




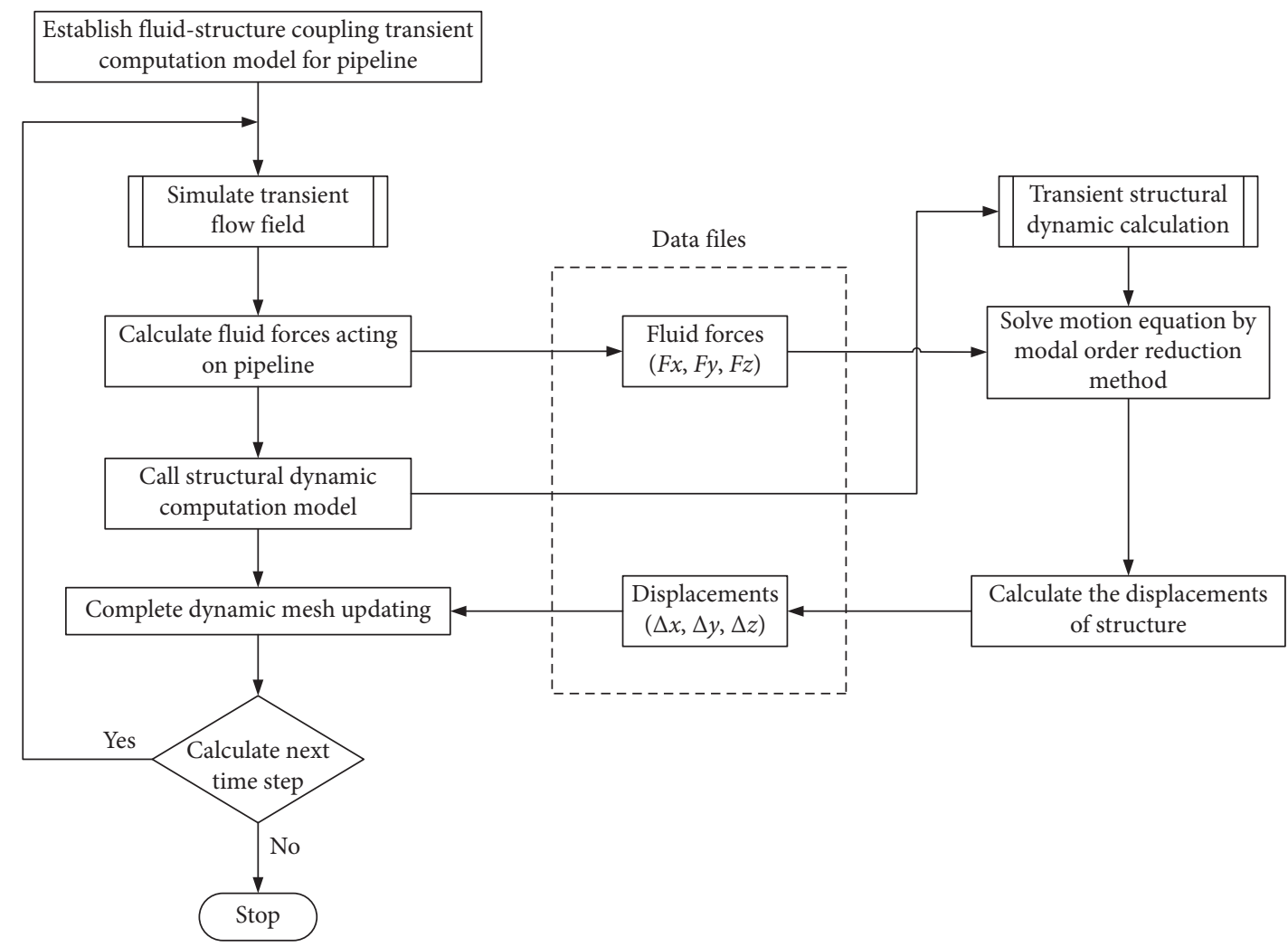

FIGURE 1: Flow chart of fluid-structure coupling transient calculation for pipeline vibration.

where $[M]$ is the mass matrix, $[C]$ is the damping matrix, $[K]$ is the stiffness matrix, $\{q\}$ is the vector of degrees of freedom, and $\{f\}$ is the external excitation vector.

Because the low-frequency vibration of pipeline is studied in this paper and the influence of higher than the fifth natural frequency on the vibration of pipeline is small, the first five modes of pipeline system are mainly considered. According to the modal order reduction, the structural motion equation of the pipeline can be simplified as follows:

$$
\left[\begin{array}{ccccc}
M_{1} & 0 & 0 & 0 & 0 \\
0 & M_{2} & 0 & 0 & 0 \\
0 & 0 & M_{3} & 0 & 0 \\
0 & 0 & 0 & M_{4} & 0 \\
0 & 0 & 0 & 0 & M_{5}
\end{array}\right]\left[\begin{array}{l}
\ddot{\eta}_{1} \\
\ddot{\eta}_{2} \\
\ddot{\eta}_{3} \\
\ddot{\eta}_{4} \\
\ddot{\eta}_{5}
\end{array}\right]+\left[\begin{array}{ccccc}
C_{1} & 0 & 0 & 0 & 0 \\
0 & C_{2} & 0 & 0 & 0 \\
0 & 0 & C_{3} & 0 & 0 \\
0 & 0 & 0 & C_{4} & 0 \\
0 & 0 & 0 & 0 & C_{5}
\end{array}\right]\left[\begin{array}{l}
\dot{\eta}_{1} \\
\dot{\eta}_{2} \\
\dot{\eta}_{3} \\
\dot{\eta}_{4} \\
\dot{\eta}_{5}
\end{array}\right]+\left[\begin{array}{ccccc}
K_{1} & 0 & 0 & 0 & 0 \\
0 & K_{2} & 0 & 0 & 0 \\
0 & 0 & K_{3} & 0 & 0 \\
0 & 0 & 0 & K_{4} & 0 \\
0 & 0 & 0 & 0 & K_{5}
\end{array}\right]\left[\begin{array}{l}
\eta_{1} \\
\eta_{2} \\
\eta_{3} \\
\eta_{4} \\
\eta_{5}
\end{array}\right]=\left[\begin{array}{l}
\phi_{1}^{\mathrm{T}}\left(x_{\mathrm{f}}, y_{\mathrm{f}}, z_{\mathrm{f}}\right) \cdot F_{\mathrm{f}} \\
\phi_{2}^{\mathrm{T}}\left(x_{\mathrm{f}}, y_{\mathrm{f}}, z_{\mathrm{f}}\right) \cdot F_{\mathrm{f}} \\
\phi_{3}^{\mathrm{T}}\left(x_{\mathrm{f}}, y_{\mathrm{f}}, z_{\mathrm{f}}\right) \cdot F_{\mathrm{f}} \\
\phi_{4}^{\mathrm{T}}\left(x_{\mathrm{f}}, y_{\mathrm{f}}, z_{\mathrm{f}}\right) \cdot F_{\mathrm{f}} \\
\phi_{5}^{\mathrm{T}}\left(x_{\mathrm{f}}, y_{\mathrm{f}}, z_{\mathrm{f}}\right) \cdot F_{\mathrm{f}}
\end{array}\right]
$$

where $M_{1}, M_{2}, M_{3}, M_{4}$, and $M_{5}$ are the first five modal masses, $C_{1}, C_{2}, C_{3}, C_{4}$, and $C_{5}$ are the first five modal damping, $K_{1}, K_{2}, K_{3}, K_{4}$, and $K_{5}$ are the first five modal stiffness, $\eta$ is the modal generalized coordinate, and $F_{\mathrm{f}}$ is the force acting on the pipeline, and the coordinates of action point are $x_{\mathrm{f}}, y_{\mathrm{f}}$, and $z_{\mathrm{f}} . \varphi_{1}, \varphi_{2}, \varphi_{3}, \varphi_{4}$, and $\varphi_{5}$ are the first five modes.

The displacement of each point in the pipeline can be obtained by the inverse transformation of the modal generalized coordinate, and its expression is as follows:

$$
q(x, y, z, t)=\left[\begin{array}{lllll}
\phi_{1} & \phi_{2} & \phi_{3} & \phi_{4} & \phi_{5}
\end{array}\right]\left\{\begin{array}{l}
\eta_{1}(t) \\
\eta_{2}(t) \\
\eta_{3}(t) \\
\eta_{4}(t) \\
\eta_{5}(t)
\end{array}\right\} .
$$

2.2. Dynamic Mesh Updating. During the transient analysis of pipeline vibration, if the fluid domain involves irregular 
movement of the pipe, dynamic mesh updating will be a difficult problem. Without proper movement of grid nodes, the calculation of transient flow field cannot be carried out. Therefore, a reliable dynamic mesh method is needed to model the flow in which the shape of the region varies with time.

2.2.1. Studied Model. The experimental apparatus of a piston air compressor piping system is shown in Figure 2. The discharge volume of the air compressor is $10 \mathrm{~m}^{3} / \mathrm{min}$, and the rated pressure is $0.8 \mathrm{MPa}$. There is an air storage tank at the outlet of the compressor to make the air supply more stable. The connection between the pipe and the supporting structure constrains the translations of the pipe, but the rotations are not fully constrained. The pipeline system consists of a main pipe and a branch pipe, and the end of the branch pipe is closed. The geometry of the computational model and the distribution of measuring points (MP) are shown in Figure 3. All the cross sections of the main and branch pipes are circular with nominal diameters $D_{\mathrm{m}}$ and $D_{\mathrm{b}}$ of $65 \mathrm{~mm}$. The standard $k$-epsilon model is used to describe the fluid flow in pipeline. Compressed air supplied to the measuring pipeline by the piston compressor is used as a fluid. The compressibility factor is calculated to be about 0.988 . Since it is close to 1 , the medium can be regarded as ideal gas. The inlet boundary condition is set to mass-flowinlet, and the outlet boundary condition is set to pressureoutlet. The pressure-velocity coupling is treated with the SIMPLE scheme, and the terms in the solution equations are discretized by the second-order upwind scheme. The hybrid initialization method is used when $t=0$ second. The time step size is 0.001 second and the time stepping method is fixed. The computational time is 8 seconds, and the pressurebased solver is chosen for the present analysis.

2.2.2. Fluid Region Division. The pipeline system includes a straight pipe, tee junction, and elbow. Their mesh sizes are different, and the rules of mesh node movement are also different. Based on the above considerations, the fluid region is divided into six parts, which are shown in Figure 4. These parts are numbered \#0 \#6.

2.2.3. Grid Motion. Figure 5 shows the $X Y$ plane of region $\# 1$. Node $K$ represents the initial position of any node in region \#1, and node $K^{\prime}$ represents the current position of node $K$ after the grid moves. Region \#1 is regarded as a slender beam, and its cross section remains flat after deformation. In the initial state, the cross section number of each node in region \#1 is given, and the calculation of cumulative displacement of each node is set up when the grid moves. In the current state, the coordinates of node $K^{\prime}$ are read, and the coordinates of the initial position $K$ are calculated according to the accumulated displacement. Then, the number of the cross sections is queried by the coordinates, and the displacement corresponding to the next time step is allocated to node $K^{\prime}$ to complete the update of

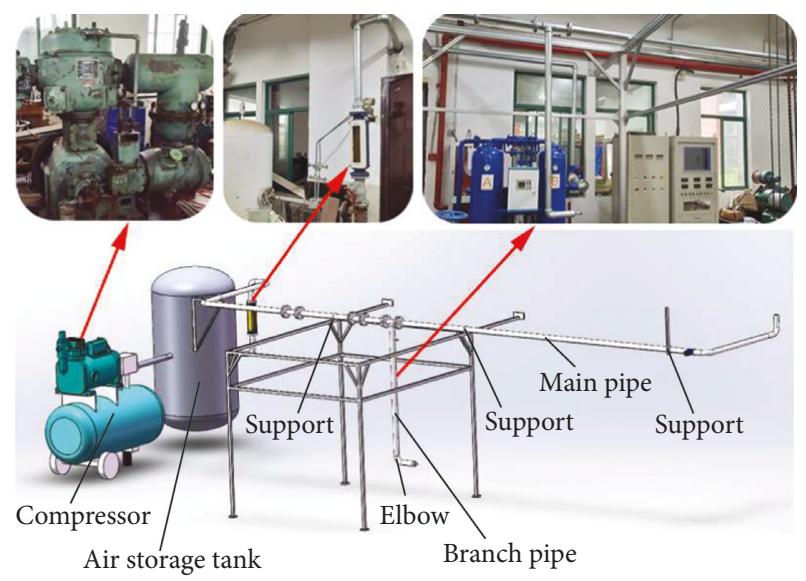

FiguRE 2: Experimental apparatus of pipeline vibration.

the motion. The grid motion algorithm developed for region \#1 applies to region \#3, region \#4, and region \#6.

Region \#2 can be considered as a rigid body, and all its nodes move with the center node as a whole.

Region \#5 is divided into several subdomains. Figure 6 shows the $X Y$ plane of region \#5. Node $P$ represents the initial position of any node in region $\# 5$, and node $P^{\prime}$ represents the current position of node $P$ after the grid moves. In the initial state, the coordinates of the center of the circle $O$ are recorded, the cross section of each node in the region 5 is numbered, and the calculation of the cumulative displacement of each node is set up when the grid moves. In the current state, the coordinates of the center of the circle $O^{\prime}$ are calculated according to the cumulative displacement. Read the coordinates of node $P^{\prime}$, calculate its azimuth angle $\alpha^{\prime}$, and judge its cross section number according to the azimuth angle. Then, the cumulative displacement is inquired according to the number, and the coordinates of the initial position $P$ are obtained. Then, the initial azimuth angle $\alpha$ of the node is calculated by using the coordinates of node $P$ and center $O$, and the initial cross section number and corresponding displacement of the next time step are obtained. The displacement is allocated to node $P^{\prime}$ to update the motion.

2.2.4. Mesh Updating Process. The process of mesh updating at the current time requires the following steps:

(1) The coordinates of all nodes and their cross section numbers in the initial state are stored, and the calculation of cumulative displacement of each node is set up.

(2) All subregions mentioned in Section 2.2.2 are accessed in turn, ensuring that all grid nodes in the fluid region are looped over. The coordinates of the nodes will be compared with the results in step (1) to determine the position of the nodes the next time.

(3) The displacement is calculated and assigned to the node according to its position, thus completing the motion updating. 


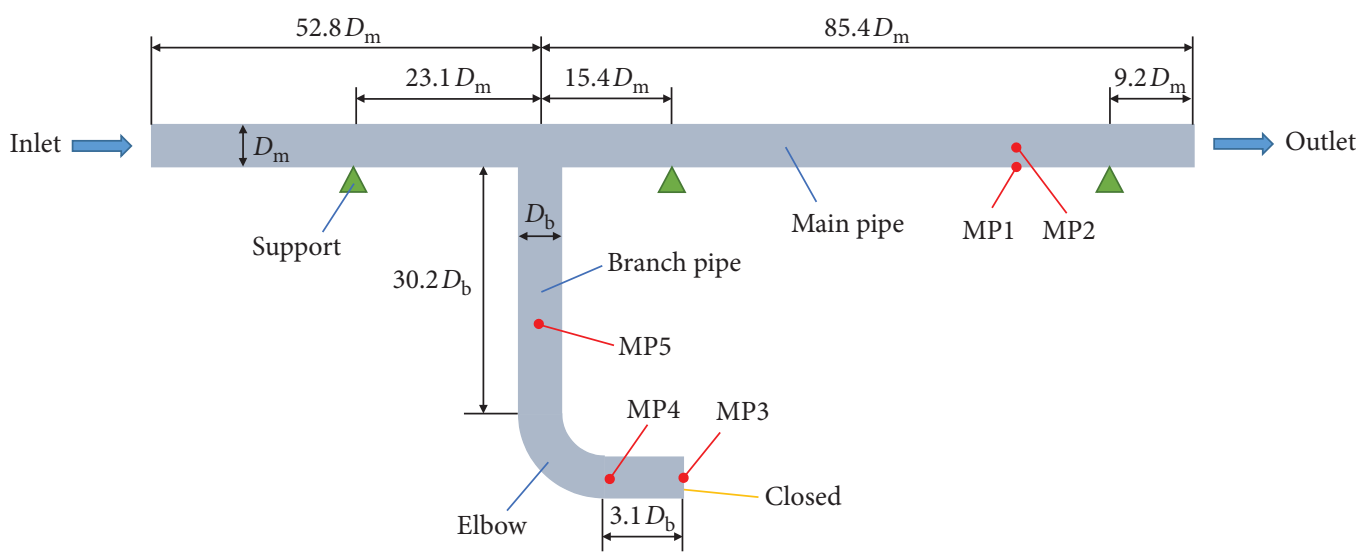

FIGURE 3: Geometry of the computational model and distribution of measuring points.

During the mesh updating process, the total number and topological relationship of the grid nodes remain unchanged. After all nodes are looped over at the current time, their coordinates are all known and can be calculated accurately at the next time. Therefore, mesh updating moves in an orderly and controllable direction. Even after tens of thousands of times of movement, the mesh after updating can still maintain high quality, thus ensuring the smooth operation of transient flow field calculation.

\section{Numerical Results}

3.1. Determination of Plane Wave Acoustic Frequency. A pulse excitation test was carried out to determine the plane wave acoustic frequencies of the pipeline. The magnitude and duration of the pulse excitation are as follows: from $0.01 \mathrm{~s}$ to $0.02 \mathrm{~s}$, the mass flow rate is $0.4 \mathrm{~kg} / \mathrm{s}$; at other times, the mass flow rate is $0.2 \mathrm{~kg} / \mathrm{s}$. The test was completed without fluid-structure coupling, and the fluid domain remained stationary. The code of pulse excitation was imported into the inlet boundary condition through UDF. The time-domain waveform under excitation is shown in Figure 7(a), and the corresponding spectrum is shown in Figure 7(b). Figure $7(\mathrm{a})$ is a decaying pulse response curve. Damping is caused by flow resistance and friction between gas molecules. It can be seen from the spectrum that the first three acoustic frequencies of plane wave are $8.25 \mathrm{~Hz}, 27.5 \mathrm{~Hz}$, and $33.0 \mathrm{~Hz}$, respectively.

\subsection{Interaction between Structural Natural Frequency and} Plane Wave Acoustic Frequency. To study the interaction between structure natural frequencies and plane wave acoustic frequencies of the pipeline, seven cases are selected. In these seven cases, only the values of modulus of elasticity have been changed to ensure that their mode shapes are the same, which is convenient for the following study. The structural natural frequencies of these seven cases are listed in Table 1. The reasons for choosing these seven cases are as follows. Firstly, the acoustic frequencies of the pipeline are obtained by the pulse excitation method. Then, Case 5 is

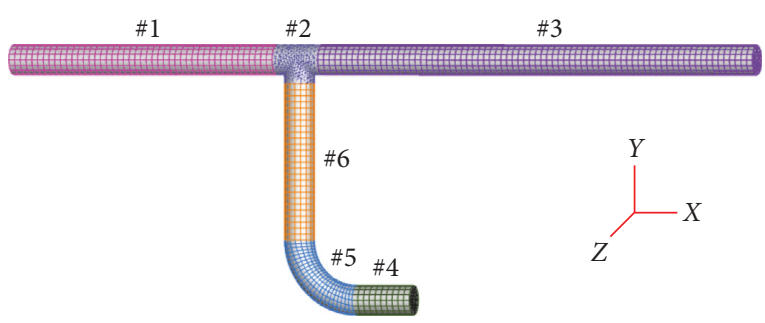

Figure 4: Region division.

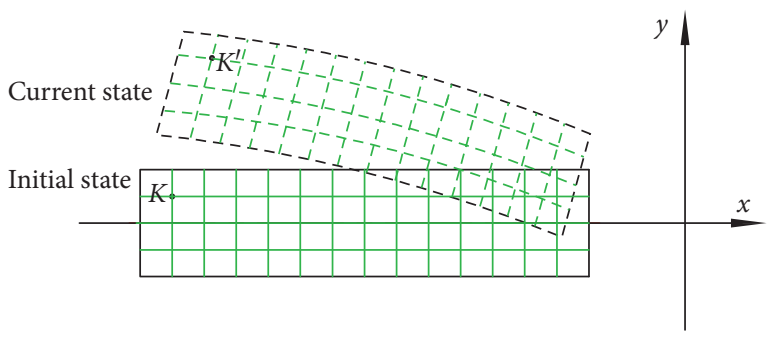

FIGURE 5: $X Y$ plane of region \#1.

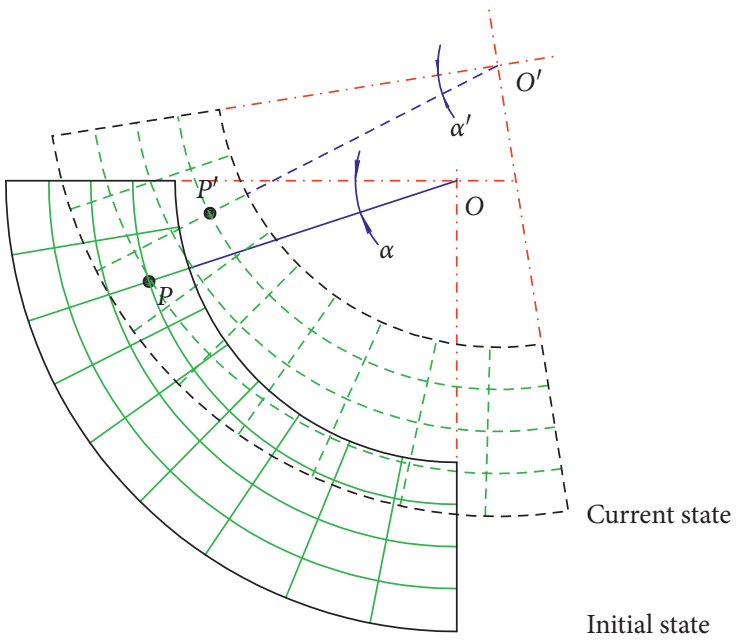

FIgURE 6: XY plane of region \#5. 


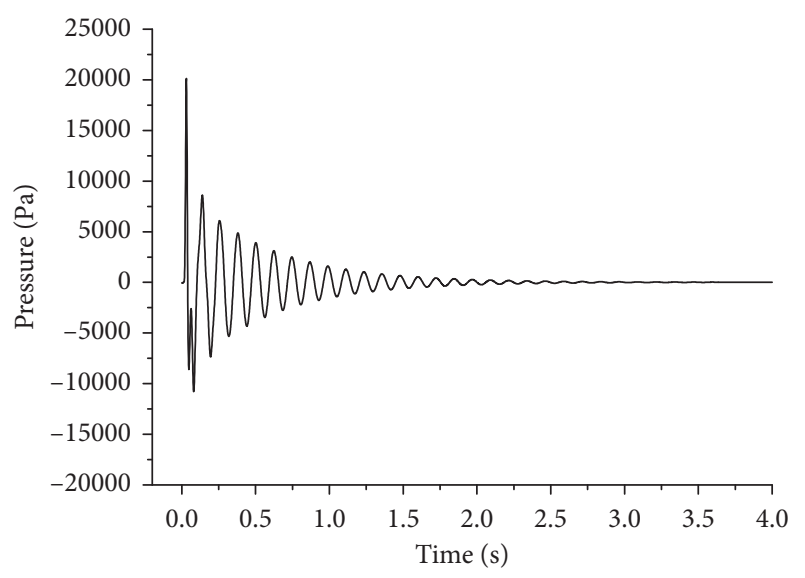

(a)

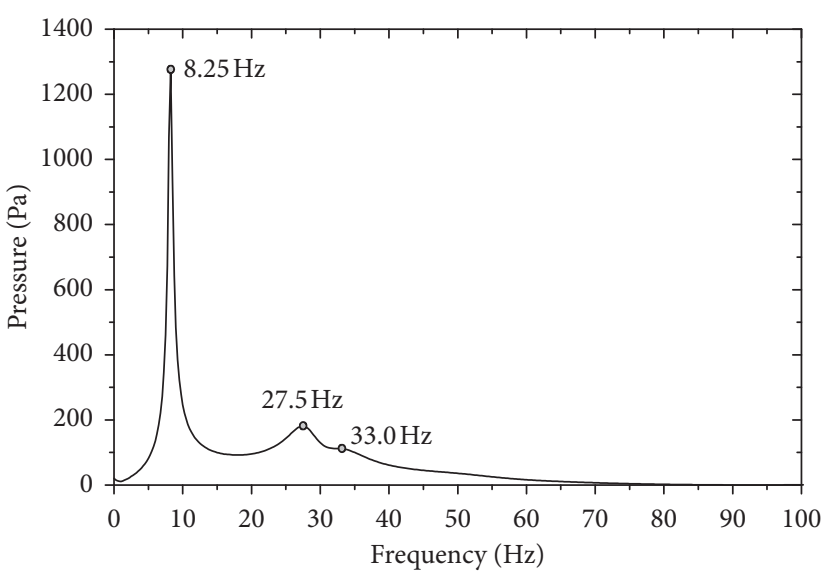

(b)

FIgURE 7: Time-domain waveform and spectrum of plane wave: (a) time-domain waveform under excitation; (b) spectrum.

TABLE 1: Structural natural frequencies of seven cases.

\begin{tabular}{lccccc}
\hline & First-order $(\mathrm{Hz})$ & Second-order $(\mathrm{Hz})$ & Third-order $(\mathrm{Hz})$ & Fourth-order $(\mathrm{Hz})$ & Fifth-order $(\mathrm{Hz})$ \\
\hline Case 1 & 6.1 & 8.3 & 13.1 & 14.8 & 2.7 \\
Case 2 & 6.5 & 8.8 & 13.8 & 16.5 & 22.1 \\
Case 3 & 6.9 & 9.3 & 14.4 & 17.6 & 23.3 \\
Case 4 & 7.4 & 10.0 & 15.3 & 20.0 & 25.0 \\
Case 5 & 8.5 & 11.5 & 17.0 & 21.0 & 28.5 \\
Case 6 & 9.0 & 12.1 & 17.8 & 26.9 & 30.1 \\
Case 7 & 12.0 & 16.2 & 22.1 & & 39.5 \\
\hline
\end{tabular}

selected to make its first-order structural natural frequency close to the first-order acoustic frequency; Case 1 is selected to make its second-order structural natural frequency close to the first-order acoustic frequency; Case 3 is selected to make its fourth-order structural natural frequency close to the frequency doubling of the first-order acoustic frequency; Case 7 is selected to make its fourth-order structural natural frequency close to the second-order acoustic frequencies, while ensuring that all frequencies are in the low-frequency range (below $40 \mathrm{~Hz}$ ). Finally, considering the continuity of the research data, Cases 2, 4, and 6 are supplemented. Through these seven cases, the potential relationship between structural natural frequencies and acoustic frequencies is explored.

The corresponding first five order mode shapes are shown in Figure 8. The first- and fourth-order mode shapes are on the $Y Z$ plane, and the second, third, and fifth-order mode shapes are on the $X Y$ plane.

Based on the fluid-structure coupling method of pipeline vibration mentioned in this paper, the transient calculation under these seven cases is carried out, respectively. The calculation results of MP4 in Case 5 are shown in Figure 9. From Figure 9(a), it can be seen that the pressure oscillation is maintained. This is due to fluid-structure coupling. When initializing, the given initial condition is not the value of the steady state, so there will be a transient response process. In this process, the pressure fluctuation of the fluid in the pipe will lead to the vibration of the pipeline structure, and the vibration of the pipeline structure will in turn affect the motion of the fluid in the pipe. This results in a continuous excitation, in which the vibration of some frequency components (Figure 9(c)) is stimulated. Therefore, the pressure oscillation can be maintained in the final steady state. As can be seen from Figure 9(b), there are three frequency components: $8.25 \mathrm{~Hz}, 17.0 \mathrm{~Hz}$, and $28.5 \mathrm{~Hz}$. However, from Figure 9(c), it can be seen that as time goes on, the frequency of $8.25 \mathrm{~Hz}$ is attenuated, and finally only two frequency components are consistent with the third- and fifth-order structural natural frequencies of Case 5. This is because the vibration displacement direction of the two structural natural frequencies coincides with the direction of the force formed by the pressure wave on the pipe wall, thus stimulating the vibration of the pipe.

It is noteworthy that the vibration displacement direction of the second-order structural natural frequency is also the same as the direction of the force formed by the pressure wave, but it is not stimulated in Figure 9(b). In order to study this phenomenon, the pressure difference of the branch pipe in $X$ and $Y$ directions is calculated, respectively. As can be seen from Figure 10, the pressure difference in the $y$ direction is much larger than that in the $x$ direction. Moreover, as can be seen from Figure 8, the vibration of the second-order structural natural frequency is in the $x$ direction of the branch pipe, the vibration of the thirdorder structural natural frequency is mainly in the $y$ direction of the main pipe and a little in the $y$-direction of the branch pipe, and the vibration of the fifth-order structural natural frequency is mainly in the $y$-direction of the branch 


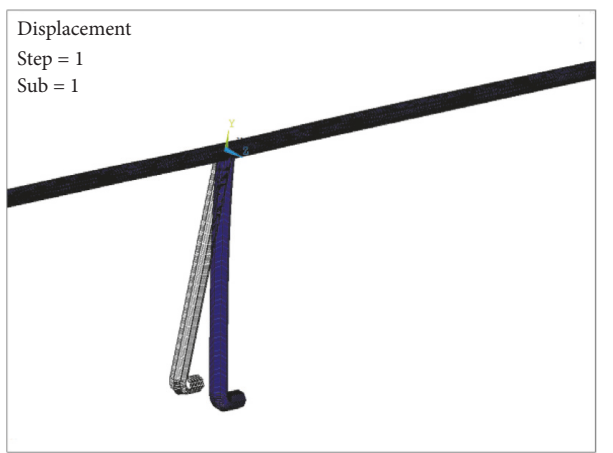

(a)

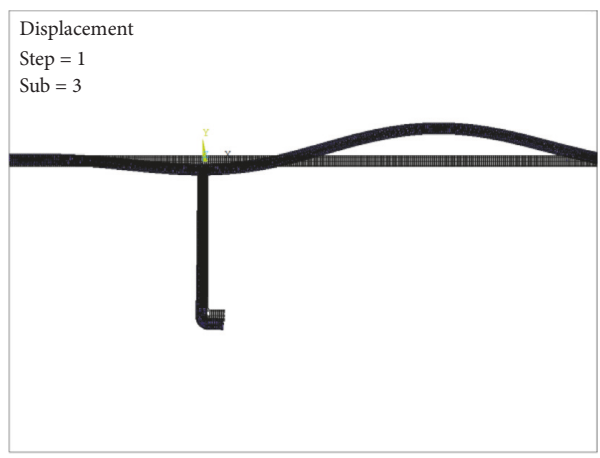

(c)

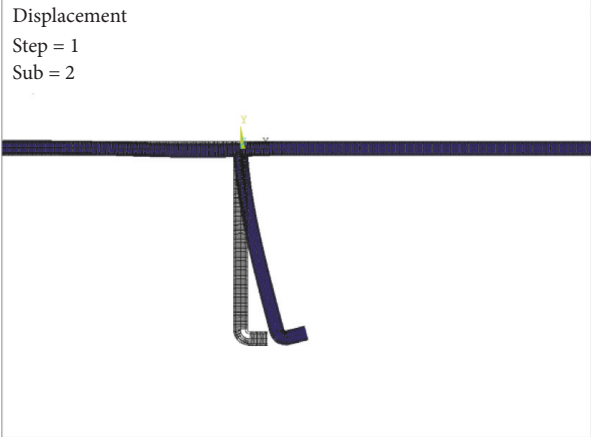

(b)

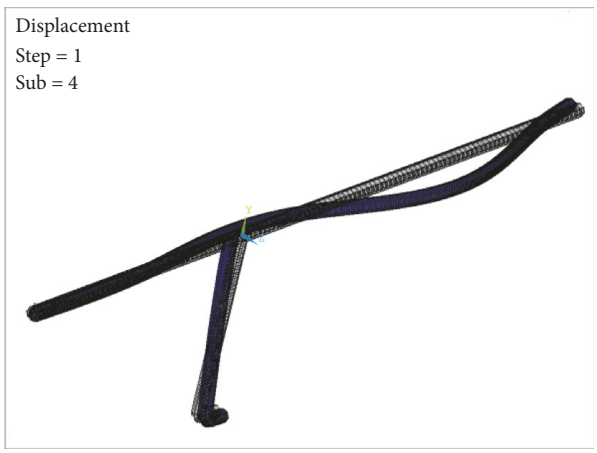

(d)

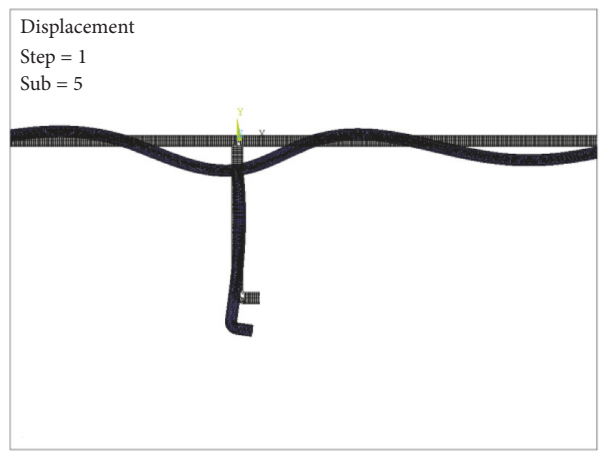

(e)

FIGURE 8: First five order mode shapes of the pipeline structure: (a) first-order mode shape, (b) second-order mode shape, (c) third-order mode shape, (d) fourth-order mode shape, and (e) fifth-order mode shape.

pipe. Therefore, the vibration of the fifth-order structural natural frequency is larger.

The analysis process of other cases is the same as above. In order to avoid redundancy, the figures of their calculation results are no longer presented in the paper. Table 2 shows the peak-peak value and main frequency components of pipeline vibration in these seven cases under fluid-structure interaction. It can be seen from the table that, in any case, the frequency components of pipeline vibration are consistent with the third- and fifth-order structural natural frequencies. Although the first-order structural natural frequency is generally considered to be the easiest to be stimulated, it is not excited after steady state because the resultant force of pressure wave acting on the $z$-direction of the pipe wall is 0 . In addition, when the vibration frequency is close to the plane wave acoustic frequency, the amplitude of the vibration increases, and when the vibration frequency is far away from the plane wave acoustic frequency, the amplitude of the vibration decreases significantly.

3.3. Effect of Different Pressures in Pipe. In order to study the effect of pressure on pipeline vibration under fluid-structure interaction, the vibration of pipeline was calculated under four different pressures. The results are shown in Figure 11. It can be seen from the figure that the greater the pressure in the pipe, the more vibration the pipe will be aroused. Moreover, in the case of nonresonance, stable inlet flow, and no external disturbance, this phenomenon also occurs. The reason is that when the pressure wave encounters the closed branch, it will produce a great impact force, which will lead to strong vibration of the pipeline structure. 


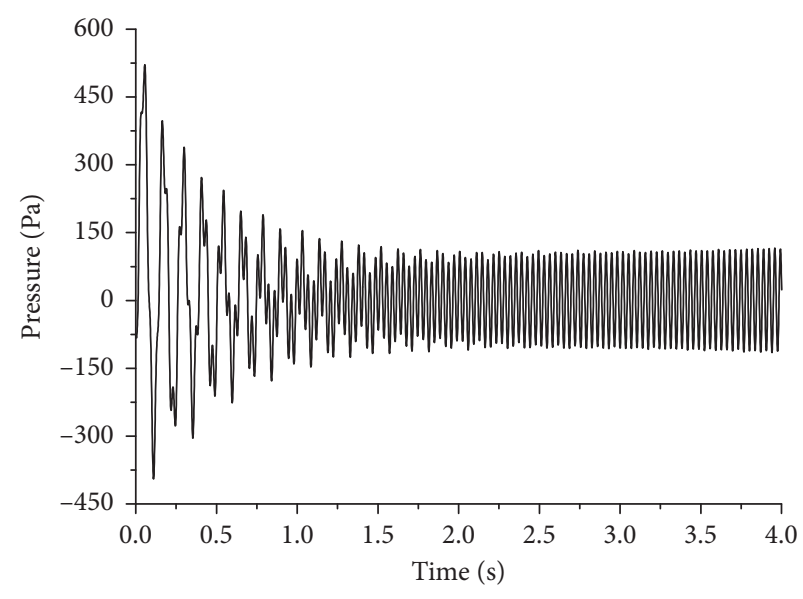

(a)

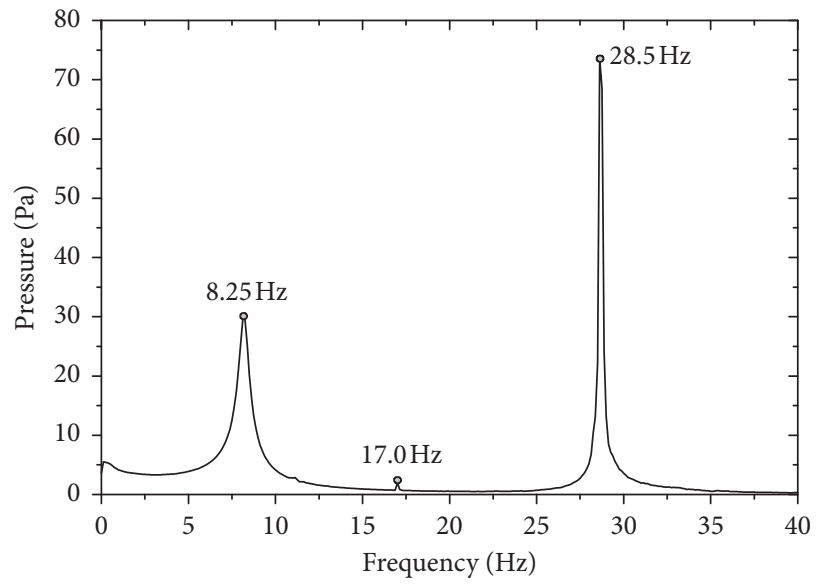

(b)

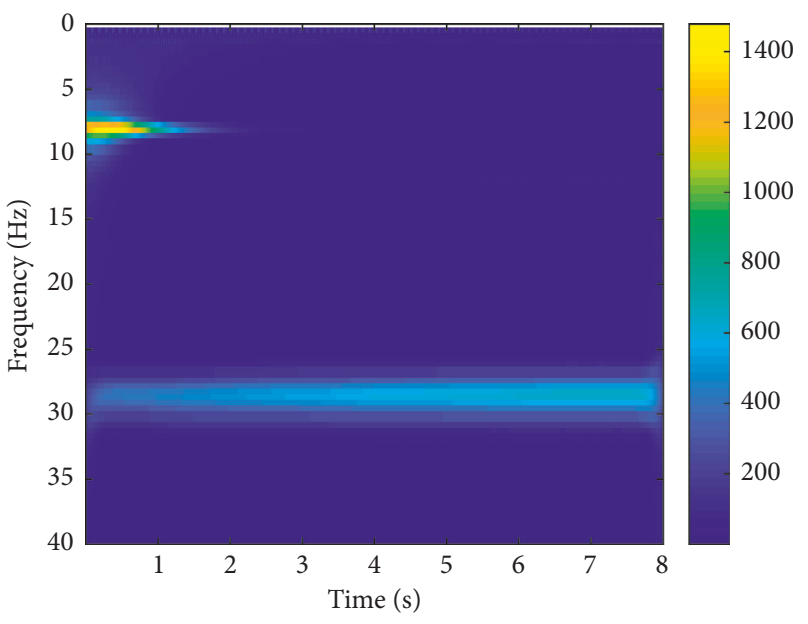

(c)

FIgURE 9: Calculation results of MP4 in Case 5: (a) time-domain waveform; (b) spectrum; (c) wavelet transform.

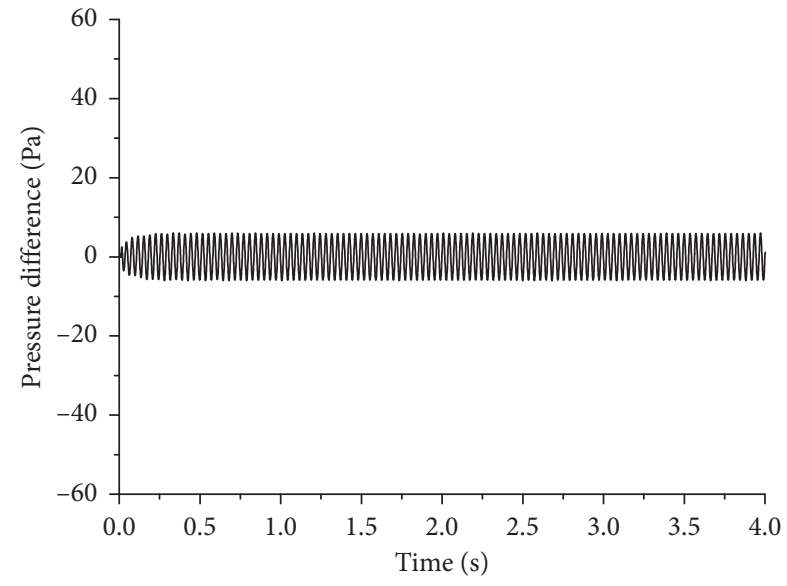

(a)

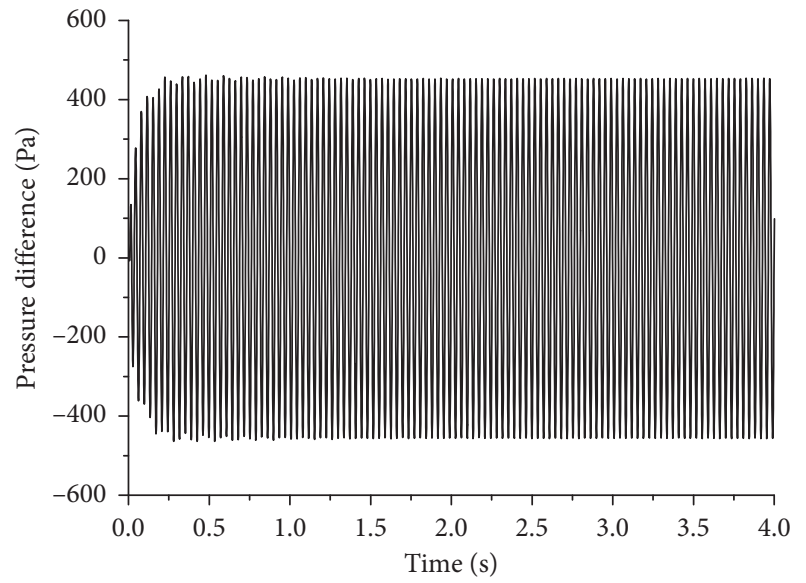

(b)

FIgURE 10: Pressure difference of the branch pipe in two directions: (a) $x$ direction; (b) $y$-direction.

3.4. Influences on Distant Downstream Pipeline. The pressure fluctuations downstream of the pipeline with and without fluid-structure coupling are given in Figure 12. It is not difficult to see that pipeline vibration has no effect on the downstream without fluid-structure coupling, but in the case of fluid-structure coupling, the vibration will propagate 
TABLE 2: Peak-peak value and main frequency components of seven cases.

\begin{tabular}{lccc}
\hline & Peak-peak value $(\mathrm{Pa})$ & \multicolumn{2}{c}{$\begin{array}{c}\text { Main frequency } \\
\text { components }(\mathrm{Hz})\end{array}$} \\
\hline Case 1 & 300 & 13.0 & 21.0 \\
Case 2 & 340 & 13.75 & 22.5 \\
Case 3 & 410 & 14.5 & 23.5 \\
Case 4 & 480 & 15.5 & 25.25 \\
Case 5 & 250 & 17.0 & 28.5 \\
Case 6 & 240 & 17.75 & 30.25 \\
Case 7 & 10 & 22.0 & 39.5 \\
\hline
\end{tabular}

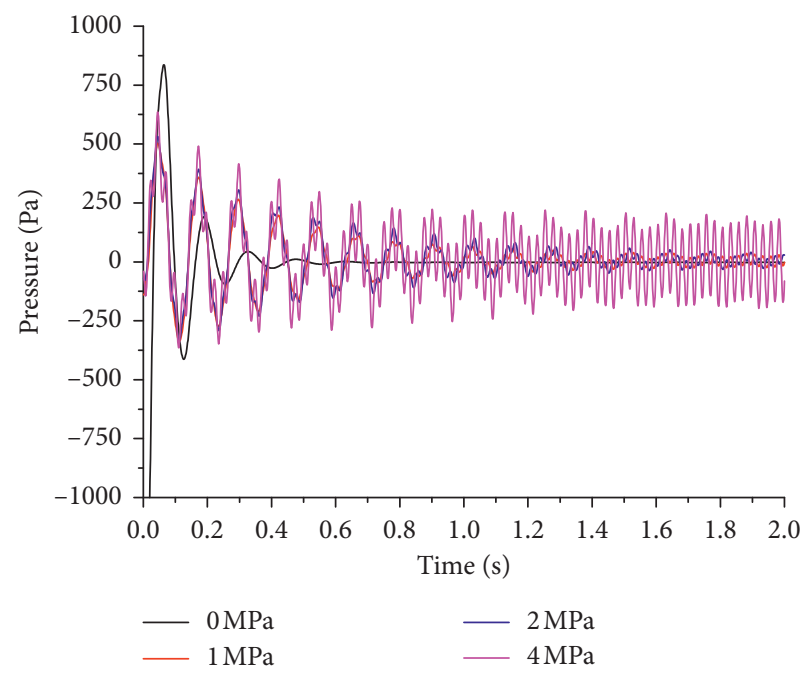

Figure 11: Vibration waveforms of MP3 in Case 7 under four different pressures.

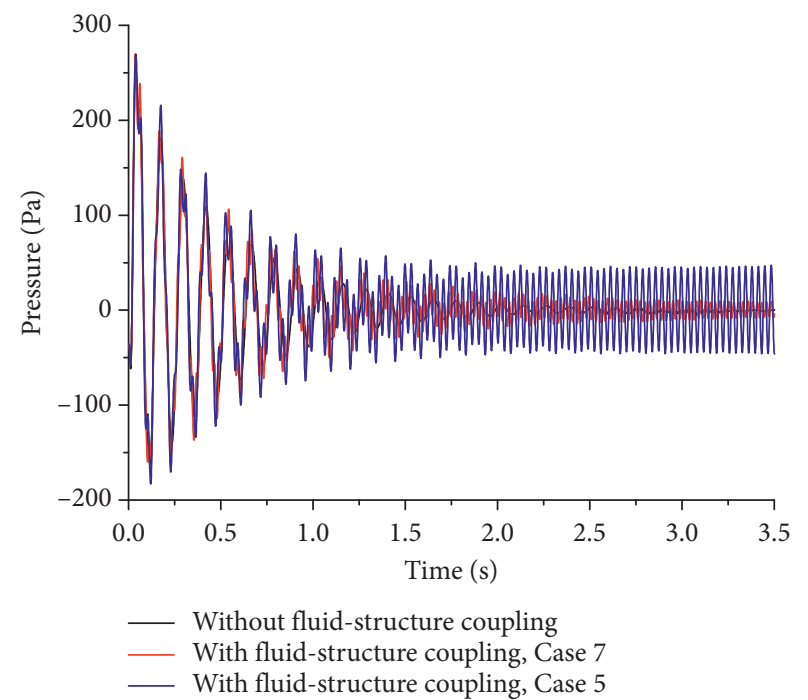

FIgURE 12: Pressure fluctuations of MP2 with and without fluidstructure coupling.

downstream. When there are tee junctions, elbows, and closed valves, it will form an exciting force in a certain direction to stimulate the vibration there.

\section{Experimental Results}

Vibration velocity sensors and pressure sensors were arranged in the measuring pipeline, and the measuring points are shown in Figure 3. Vibration signals from the vibration velocity sensors and pressure signals from the pressure sensors were recorded on PC data acquisition software by using a data acquisition board. The schematic of measuring system is shown in Figure 13. The sampling frequency was $1000 \mathrm{~Hz}$, and the sampling time was $8 \mathrm{~s}$. The time-domain data were transformed into the frequencydomain data by fast-Fourier transform (FFT).

4.1. Determination of Structural Natural Frequencies. An excitation test was carried out to determine structural natural frequencies of the pipeline. A force hammer is used to strike the pipeline quickly, which is equivalent to a pulse excitation to the pipeline structure. Although the magnitude and duration of the excitation are unknown, it does not affect the acquisition of structural natural frequencies. They can be obtained by spectrum analysis of the measured vibration signals under excitation. The time-domain waveform of MP1 under excitation is shown in Figure 14(a), and the corresponding spectrum is shown in Figure 14(b). It can be seen from the spectrum that there is a strongly dominant frequency of $15.1 \mathrm{~Hz}$, which is a certain order structural natural frequency. Using the same method, excitation tests were also carried out at other measuring points. In order to avoid redundancy, the figures of excitation test results at other measuring points are no longer given in the paper. Table 3 shows the structural natural frequencies measured at all measuring points. As can be seen from Table 3, the first five structural natural frequencies of the pipeline system are $4.6 \mathrm{~Hz}, 8.4 \mathrm{~Hz}, 15.1 \mathrm{~Hz}, 21.4 \mathrm{~Hz}$, and $25.9 \mathrm{~Hz}$, respectively.

4.2. Vibration Comparison under Different Pressures. Vibration measurements under six different pipeline pressures were carried out. The six kinds of pipeline pressure are $0.1 \mathrm{MPa}, 0.2 \mathrm{MPa}, 0.3 \mathrm{MPa}, 0.4 \mathrm{MPa}, 0.5 \mathrm{MPa}$, and $0.56 \mathrm{MPa}$, respectively. Vibration data are obtained and analyzed at each measuring point under each kind of pipeline pressure.

Figure 15 shows the time-domain waveform and spectrum of MP4 at a pressure of $0.2 \mathrm{MPa}$. As can be seen from Figure 15(b), the main frequency components are $16.5 \mathrm{~Hz}$ and $24.9 \mathrm{~Hz}$. By comparing them with the structural natural frequencies described in Section 4.1, it can be seen that they are consistent with the third- and fifth-order structural natural frequencies. In addition, by comparing the time histories of experimental measurement and numerical calculation, it can be seen that Figure 9(a) has a transient response process, while Figure 15(a) does not. The reasons are as follows. In numerical calculation, the initial condition given for initialization is not the value of the steady state, so there will be a transient response process. In this process, the pressure fluctuation of the fluid in the pipe will lead to the vibration of the pipeline structure, and the vibration of the pipeline structure will in turn affect the motion of the fluid in the pipe. Vibration that is inconsistent with the direction of 


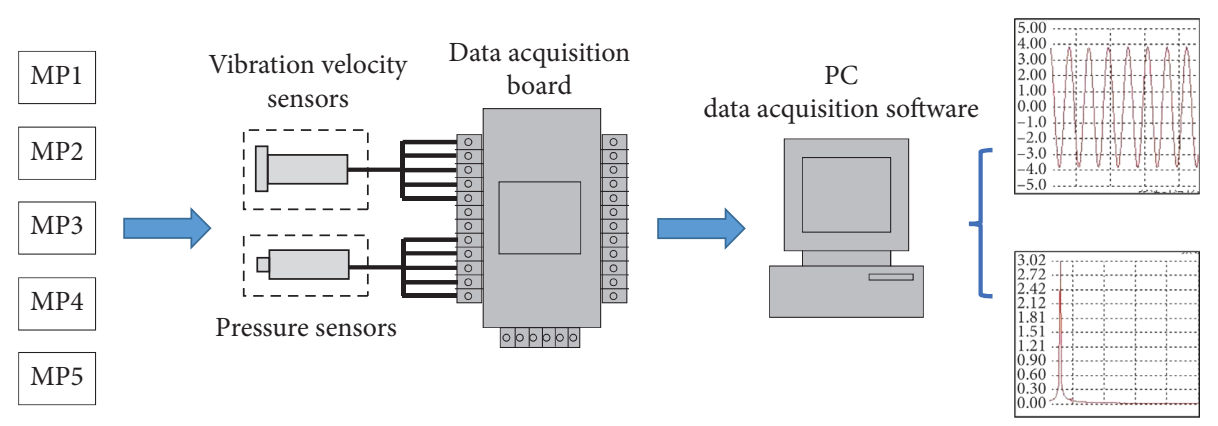

FIgURE 13: Schematic of the measuring system.

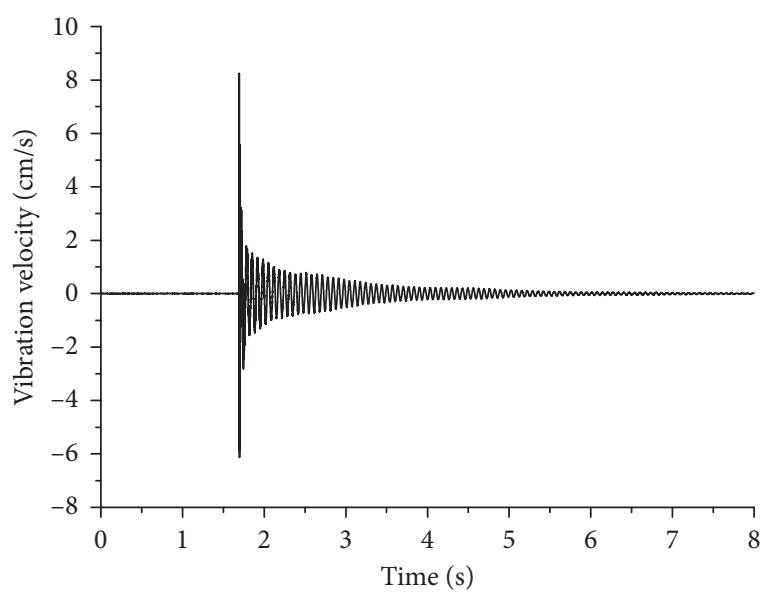

(a)

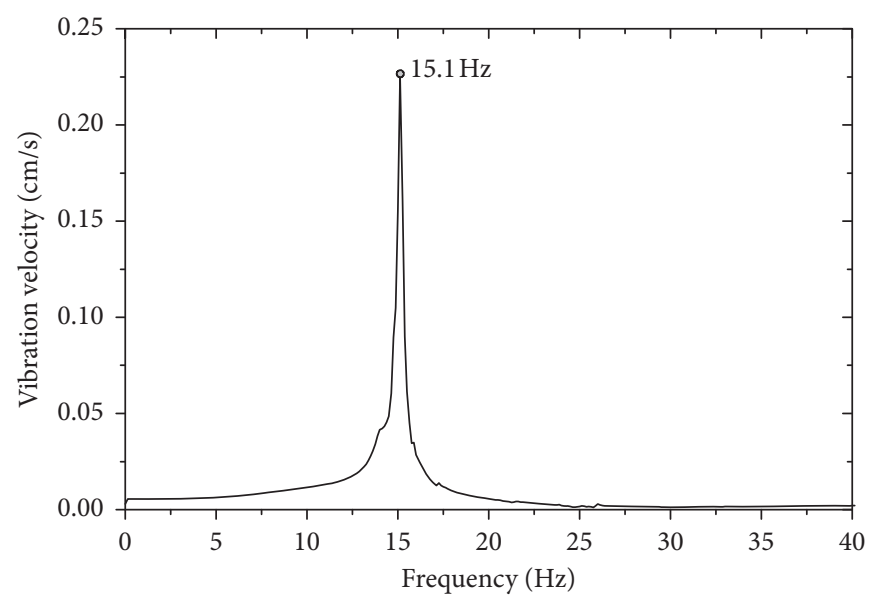

(b)

FIGURE 14: Time-domain waveform and spectrum of MP1: (a) time-domain waveform under excitation; (b) spectrum.

TABLE 3: Structural natural frequencies measured at all measuring points.

\begin{tabular}{|c|c|c|c|c|c|}
\hline & First-order $(\mathrm{Hz})$ & Second-order $(\mathrm{Hz})$ & Third-order $(\mathrm{Hz})$ & Fourth-order $(\mathrm{Hz})$ & Fifth-order $(\mathrm{Hz})$ \\
\hline MP1 & - & - & 15.1 & - & - \\
\hline MP2 & - & - & - & 21.4 & - \\
\hline MP3 & - & 8.4 & - & - & - \\
\hline MP4 & 4.6 & - & - & - & 25.9 \\
\hline MP5 & 4.6 & - & - & - & 25.9 \\
\hline
\end{tabular}

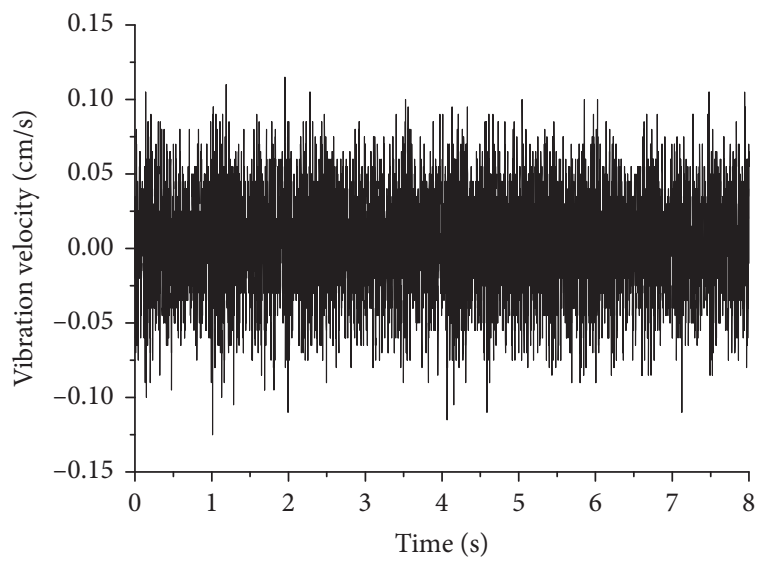

(a)

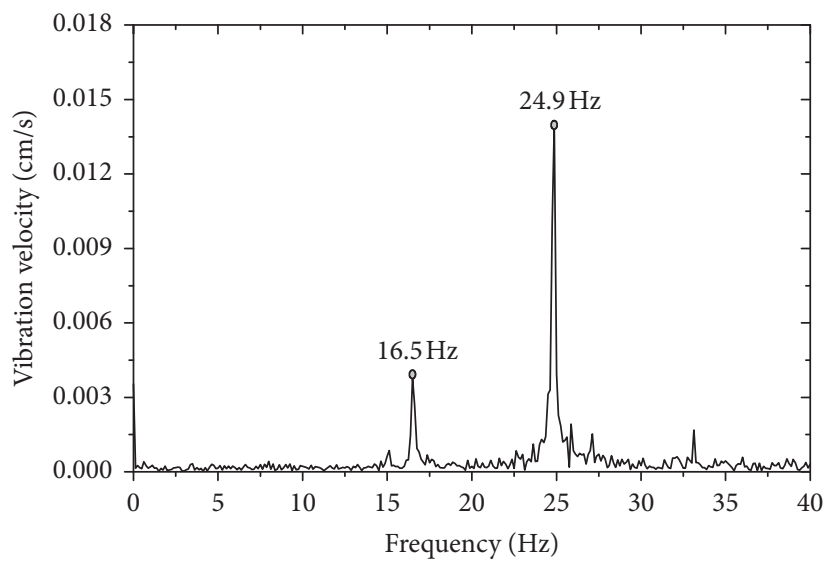

(b)

Figure 15: Time-domain waveform and spectrum of MP4, 0.2 MPa: (a) time-domain waveform; (b) spectrum. 
TABLE 4: Peak-peak value and main frequency components of MP4 under different pressures.

\begin{tabular}{lccr}
\hline & Peak-peak value $(\mathrm{cm} / \mathrm{s})$ & \multicolumn{2}{c}{$\begin{array}{c}\text { Main frequency } \\
\text { components } \\
(\mathrm{Hz})\end{array}$} \\
\hline $0.1 \mathrm{MPa}$ & 0.08 & 16.6 & 24.9 \\
$0.2 \mathrm{MPa}$ & 0.12 & 16.5 & 24.9 \\
$0.3 \mathrm{MPa}$ & 0.15 & 16.5 & 24.8 \\
$0.4 \mathrm{MPa}$ & 0.18 & 16.4 & 24.6 \\
$0.5 \mathrm{MPa}$ & 0.20 & 16.4 & 24.6 \\
$0.56 \mathrm{MPa}$ & 0.21 & 16.4 & 24.6 \\
\hline
\end{tabular}

the force generated by the pressure wave will attenuate to 0 , and vibration that is consistent with the direction of the force generated by the pressure wave will maintain and tend to oscillate stably. However, in the experiment, the vibration measurement is carried out after the compressor is opened and operated for a period of time, so there is no transient process. The steady-state experiment is similar to the steadystate oscillation process after 3 seconds in Figure 9(a). It should be noted that the experiment results also contain noise signals. This steady-state oscillation process also needs to be solved by the transient solution method. The purpose of this paper is to study the fluid-structure coupling vibration of the pipeline under steady oscillatory state. Therefore, by comparing the experimental results with the numerical results under steady oscillatory state, it can be seen that the vibration frequencies are consistent with the third- and fifth-order structural natural frequencies.

Table 4 shows the peak-peak value and main frequency components of MP4 under different pressures. It can be seen from the table that no matter how much pipe pressure is, the frequencies of pipeline vibration are the structural natural frequencies, which is consistent with the results of numerical calculation in Section 3.2. Furthermore, the higher the pipe pressure, the greater the vibration, which is in agreement with the results of numerical calculation in Section 3.3. The same conclusions are reached at other measuring points.

\section{Conclusions}

(1) The fluid-structure coupling method proposed is reasonable and reliable by comparing numerical results with experimental results and can be applied to engineering.

(2) Through the fluid-structure coupling calculation, it is found that the pressure fluctuation and the vibration of pipeline structure will occur when the inlet flow is stable and there is no external disturbance. The vibration frequencies are consistent with the third- and fifth-order structural natural frequencies, and the first-order structural natural frequency is not excited due to the direction of force.

(3) The higher the pressure in the pipe, the greater the fluid-structure coupling vibration. It is useless to add accumulators in the high-pressure pipeline. Although the inlet flow is steady, there will still be vibration in the pipeline.
(4) The fluid-structure coupling vibration not only occurs in the studied pipeline but also propagates to distant downstream pipeline. When there are tee junctions, elbows, and closed valves, it will form an exciting force in a certain direction to stimulate the vibration there.

\section{Data Availability}

The data used to support the findings of this study are available from the corresponding author upon request.

\section{Conflicts of Interest}

The authors declare that they have no conflicts of interest.

\section{Acknowledgments}

This work was supported by the National Key Research and Development Program of China (no. 2016YFC0801200).

\section{References}

[1] E. Skudrzyk, The Foundations of Acoustics, Springer-Verlag, Berlin, Germany, 1971.

[2] S. Hayama, Y. Mohri, and T. Watanabe, "Resonant amplitudes of pressure pulsation in pipelines: 1st report, resonant amplitudes in case of a single sinusoidal flow input," Bulletin of JSME, vol. 20, no. 146, pp. 955-962, 1977.

[3] J. F. T. Maclaren, A. B. Tramschek, A. Sanjines, and O. F. Pastrana, "A comparison of numerical solutions of the unsteady flow equations applied to reciprocating compressor systems," Journal of Mechanical Engineering Science, vol. 17, no. 5, pp. 271-279, 1975.

[4] C. Durant, G. Robert, P. J. T. Filippi, and P.-O. Mattei, "Vibroacoustic response of a thin cylindrical shell excited by a turbulent internal flow: comparison between numerical prediction and experimentation," Journal of Sound and Vibration, vol. 229, no. 5, pp. 1115-1155, 2000.

[5] M. P. Païdoussis and N. T. Issid, "Dynamic stability of pipes conveying fluid," Journal of Sound and Vibration, vol. 33, no. 3, pp. 267-294, 1974.

[6] S. S. Chen, "Vibrations of continuous pipes conveying fluid," in Flow-Induced Structural Vibrations, pp. 663-675, Springer, Berlin, Germany, 1974.

[7] T. Irie, G. Yamada, and I. Takahashi, "Vibration and stability of a non-uniform Timoshenko beam subjected to a follower force," Journal of Sound and Vibration, vol. 70, no. 4, pp. 503-512, 1980.

[8] M. W. Lesmez, D. C. Wiggert, and F. J. Hatfield, "Modal analysis of vibrations in liquid-filled piping systems," Journal of Fluids Engineering, vol. 112, no. 3, pp. 311-319, 1990.

[9] D. C. Wiggert, R. S. Otwell, and F. J. Hatfield, "The effect of elbow restraint on pressure transients," Journal of Fluids Engineering, vol. 107, no. 3, pp. 402-406, 1985.

[10] D. C. Wiggert, F. J. Hatfield, and S. Stuckenbruck, "Analysis of liquid and structural transients in piping by the method of characteristics," Journal of Fluids Engineering, vol. 109, no. 2, pp. 161-165, 1987.

[11] W. Erath, B. Nowotny, and J. Maetz, "Modelling the fluid structure interaction produced by a waterhammer during shutdown of high-pressure pumps," Nuclear Engineering and Design, vol. 193, no. 3, pp. 283-296, 1999. 
[12] A. S. Tijsseling and C. S. W. Lavooij, "Waterhammer with fluid-structure interaction," Applied Scientific Research, vol. 47, no. 3, pp. 273-285, 1990.

[13] B. Sreejith, K. Jayaraj, N. Ganesan, C. Padmanabhan, P. Chellapandi, and P. Selvaraj, "Finite element analysis of fluid-structure interaction in pipeline systems," Nuclear Engineering and Design, vol. 227, no. 3, pp. 313-322, 2004.

[14] M. T. Pittard, R. P. Evans, R. D. Maynes, and J. D. Blotter, "Experimental and numerical investigation of turbulent flow induced pipe vibration in fully developed flow," Review of Scientific Instruments, vol. 75, no. 7, pp. 2393-2401, 2004.

[15] F. Menter, P. Sharkey, S. Yakubov, and M. Kuntz, "Overview of fluid-structure coupling in ANSYS-CFX," in 25th International Conference on Offshore Mechanics and Arctic Engineering, Hamburg, Germany, June 2006.

[16] A. E. Vardy, D. Fan, and A. S. Tijsseling, "Fluid-structure interaction in a T-piece pipe," Journal of Fluids and Structures, vol. 10, no. 7, pp. 763-786, 1996.

[17] A. S. Tijsseling, A. E. Vardy, and D. Fan, "Fluid-structure interaction and cavitation in a single-elbow pipe system," Journal of Fluids and Structures, vol. 10, no. 4, pp. 395-420, 1996.

[18] A. S. Tijsseling, "An overview of fluid-structure interaction experiments in single-elbow pipe systems," Journal of Zhejiang University-SCIENCE A, vol. 20, no. 4, pp. 233-242, 2019.

[19] S. Ziada, K. W. Mclaren, and Y. Li, "Flow-acoustic coupling in T-junctions: effect of T-junction geometry," Journal of Pressure Vessel Technology, vol. 131, no. 4, article 041302, 2009.

[20] G. B. Duan, Z. M. Liu, G. L. Chen, S. G. Hu, and J. Zhao, "Experimental investigation of gas-solid two-phase flow in Y-shaped pipeline," Advanced Powder Technology, vol. 21, no. 4 , pp. $468-476,2010$. 


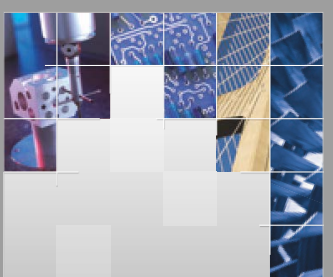

\section{Enfincering}
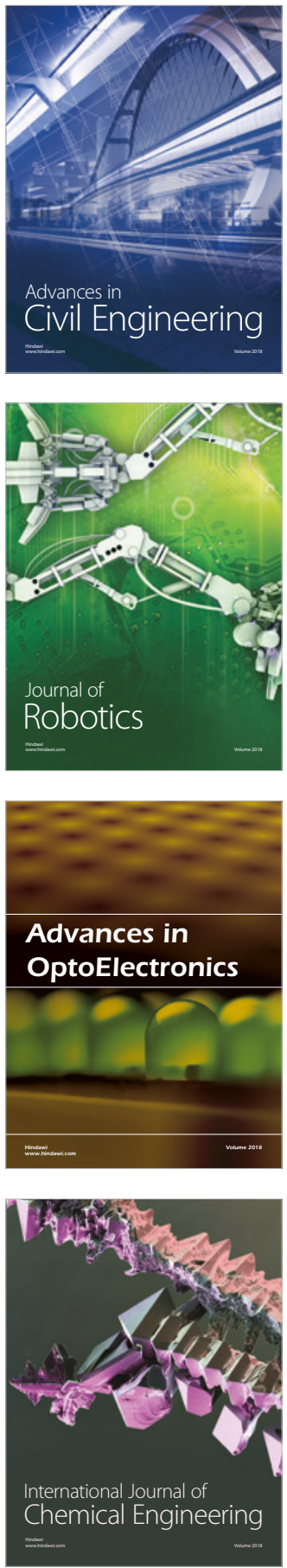

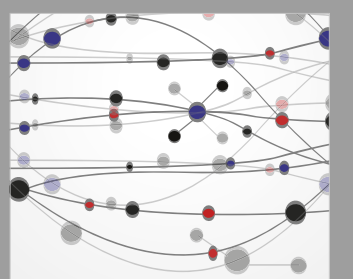

\section{Rotating \\ Machinery}

The Scientific World Journal

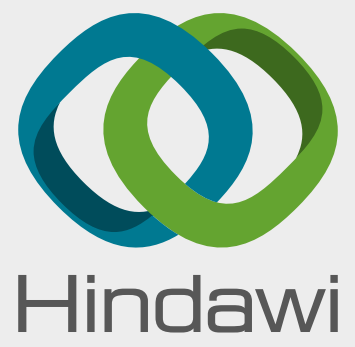

Submit your manuscripts at

www.hindawi.com
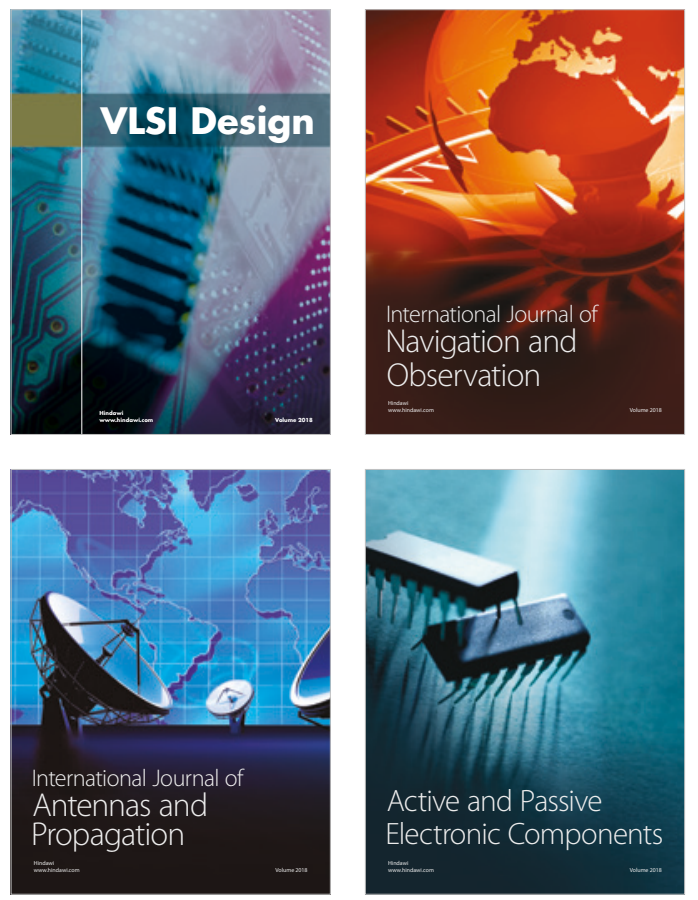
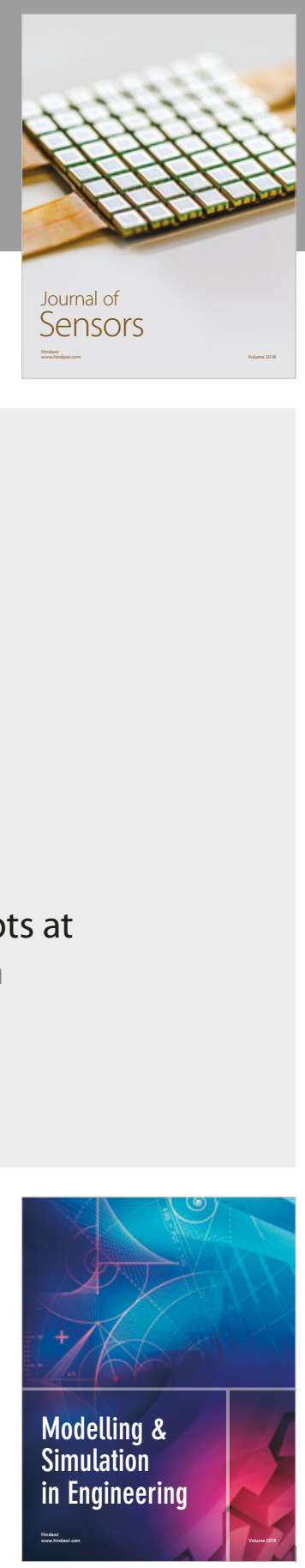

\section{Advances \\ Multimedia}
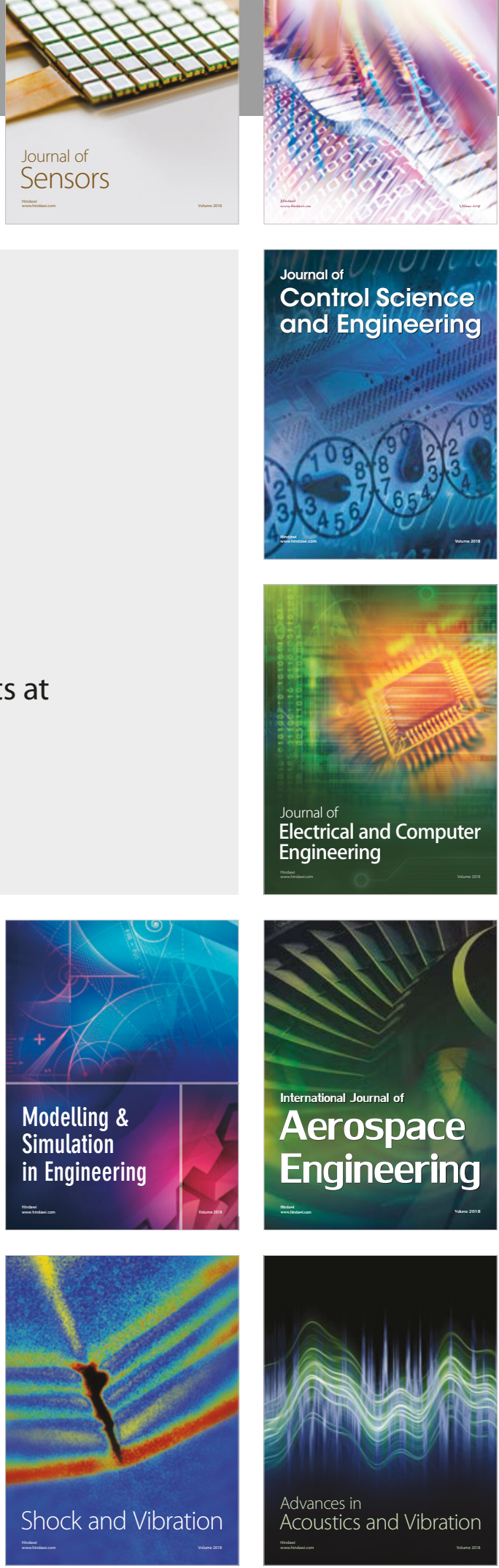